\title{
Ciprofloxacin resistance in community- and hospital-acquired Escherichia coli urinary tract infections: a systematic review and meta-analysis of observational studies
}

Oyebola Fasugba ${ }^{{ }^{*}}$, Anne Gardner ${ }^{1}$, Brett G. Mitchell ${ }^{1,2}$ and George Mnatzaganian ${ }^{3}$

\begin{abstract}
Background: During the last decade the resistance rate of urinary Escherichia coli (E. coli) to fluoroquinolones such as ciprofloxacin has increased. Systematic reviews of studies investigating ciprofloxacin resistance in community- and hospital-acquired E. coli urinary tract infections (UTI) are absent. This study systematically reviewed the literature and where appropriate, meta-analysed studies investigating ciprofloxacin resistance in community- and hospital-acquired E. coli UTIs.
\end{abstract}

Methods: Observational studies published between 2004 and 2014 were identified through Medline, PubMed, Embase, Cochrane, Scopus and Cinahl searches. Overall and sub-group pooled estimates of ciprofloxacin resistance were evaluated using DerSimonian-Laird random-effects models. The $\mathrm{I}^{2}$ statistic was calculated to demonstrate the degree of heterogeneity. Risk of bias among included studies was also investigated.

Results: Of the identified 1134 papers, 53 were eligible for inclusion, providing 54 studies for analysis with one paper presenting both community and hospital studies. Compared to the community setting, resistance to ciprofloxacin was significantly higher in the hospital setting (pooled resistance $0.38,95 \% \mathrm{Cl} 0.36-0.41$ versus $0.27,95 \% \mathrm{Cl}$ 0.24-0.31 in community-acquired UTIs, $P<0.001$ ). Resistance significantly varied by region and country with the highest resistance observed in developing countries. Similarly, a significant rise in resistance over time was seen in studies reporting on community-acquired E. coli UTI.

Conclusions: Ciprofloxacin resistance in E. coli UTI is increasing and the use of this antimicrobial agent as empirical therapy for UTI should be reconsidered. Policy restrictions on ciprofloxacin use should be enhanced especially in developing countries without current regulations.

Keywords: Antimicrobial resistance, Escherichia coli, Urinary tract infection, Systematic review, Meta-analysis

\section{Background}

Urinary tract infections (UTI) are one of the most frequent bacterial infections affecting people both in the community and in hospitals [1]. It is estimated that about 150 million people per annum are diagnosed with UTI worldwide [2]. A recent World Health Organisation (WHO) report on antimicrobial resistance (AMR) surveillance specified nine bacteria of international concern which are responsible for

\footnotetext{
* Correspondence: oyebola.fasugba@myacu.edu.au

${ }^{1}$ Faculty of Health Sciences, Australian Catholic University, 223 Antill Street, Watson, Australian Capital Territory 2602, Australia

Full list of author information is available at the end of the article
}

some of the most common infections in community and hospital settings [3]. Escherichia coli (E. coli), the pathogen most often implicated in UTIs, is listed as one of the nine. In all six WHO regions (Africa, Americas, Eastern Mediterranean, European, South-East Asia and Western Pacific) high rates of antimicrobial resistance have been observed in this pathogen [3].

Ciprofloxacin is the most commonly prescribed fluoroquinolone for UTIs because it is available in oral and intravenous preparations [4]. It is well absorbed from the gastrointestinal tract after oral administration. It also has a documented safety profile, broad Gram 
negative organism coverage and high urinary excretion rate [4]. During the last decade the resistance rate of $E$. coli to fluoroquinolones such as ciprofloxacin has increased [5]. A 10 year analysis of urinary E. coli specimens in Switzerland, found an increasing trend in resistance to ciprofloxacin, from 1.8 to 15.9 \% [6]. Fluoroquinolones are ranked as one of four of the highest priority critically important antimicrobials [7] as they have an important role in the treatment of more severe infections, such as septicaemia. Therefore resistance to fluoroquinolones can have serious clinical consequences. They are one of few available therapies for serious Salmonella spp. and E.coli infections [5]. Resistance to fluoroquinolones emerges quickly, and this is likely to be related to the biology of resistance as well as a direct response to drug pressure [8]. They should therefore be used with caution and reserved for severe infections, and preceded by antimicrobial susceptibility testing of the bacteria involved [5]. The most recent Infectious Diseases Society of America (IDSA) guidelines recommend that fluoroquinolones should be reserved for important uses due to their propensity for ecological unfavorable effects of antimicrobial therapy such as the selection of drug-resistant pathogens and colonisation or infection with multidrug-resistant organisms [9].

Recent prescribing guidelines recommend reserving ciprofloxacin use for more severe infections and resistance to this agent is increasing prompting further research in this area $[6,10,11]$. Published quantitative syntheses of overall ciprofloxacin-resistant $E$. coli UTI prevalence and incidence in hospital and community settings are absent. This systematic review of observational studies therefore aims to compare ciprofloxacin resistance in both settings. Knowledge about ciprofloxacin resistance in community- and hospital-acquired E. coli UTIs will provide information for control of resistant pathogens. This review also has the potential to provide a basis for which future interventions can be evaluated. The findings will, in addition, make available information on ciprofloxacin resistance in various regions of the world providing some evidence for further regulatory control of ciprofloxacin use globally.

\section{Methods}

\section{Protocol and registration}

The protocol for conducting this review has been registered and can be accessed on the International prospective register of systematic reviews (PROSPERO) (available at http://www.crd.york.ac.uk/prospero/ with registration number: CRD42014014473). Prior to registration, the protocol was reviewed by a reviewer external to the study team. Ethics approval was not sought as this review synthesized data from published studies for which approval had already been obtained.

\section{Search strategy}

We conducted a systematic review of observational (cross sectional, cohort and case control) studies published in the last 11 years (2004-2014) reporting on ciprofloxacin resistance in community- and hospital-acquired E. coli UTIs. This time limit is based on changes in the microbiology and epidemiology of antimicrobial resistant pathogens which occurred in the past decade with subsequent changes in treatment regimens and patient outcomes [12]. Reporting of this review complied with the preferred reporting items for systematic reviews and meta-analyses (PRISMA) [13].

The electronic bibliographic databases MEDLINE/ PubMed, EMBASE, Cochrane, CINAHL and Scopus were searched. Searches were conducted for words in the title or abstract or within the full text of the papers. These included both keywords only and keywords with medical subject headings (MeSH) using the search terms 'resistance,' urinary tract infection' and 'Escherichia coli' from 1st January 2004 to 31st December 2014 (see Additional file 1). The reference lists of papers identified from the electronic databases were hand-searched for additional papers.

\section{Inclusion and exclusion criteria}

Papers were included if they reported prevalence or incidence rates of ciprofloxacin resistance in community- or hospital-acquired E. coli UTIs. Papers reporting on urinary $E$. coli ciprofloxacin susceptibility in which resistance rate could be calculated were also included. We included papers involving adults and/or children. Only peer reviewed manuscripts were considered. Grey material which includes unpublished literature, conference abstracts, letters to editors, newsletters and reports were excluded. Nonpeer reviewed literature were also excluded. Papers written in languages other than English were also excluded. In addition, papers not clearly specifying the setting (hospital-acquired or community-acquired); drug (ciprofloxacin) or sample (urine) were excluded. Papers that focused on specific sub-populations (e.g. diabetics and patients with recurrent UTI) were also excluded as these did not represent the general population. This review included only papers that used the Centers for Disease Control and Prevention (CDC) definition of microbiologically confirmed UTI ( $\geq 10^{5}$ colony forming unit/ml) [14].

\section{Definitions}

For the purpose of this review, a study was defined as all data from a published paper with the only distinction being 'hospital' or 'community' setting. Therefore, if a single paper meeting the eligibility criteria reported data on both settings, they were included as two separate studies.

Community-acquired UTI was defined as positive samples obtained from (i) outpatient clinics; (ii) general practice (GP) clinics; (iii) emergency departments; (iv) within 
$48 \mathrm{~h}$ of hospital admission or (v) from nursing homes or residential aged care facilities [15-17].

Hospital-acquired UTI was defined as positive samples obtained (i) after $48 \mathrm{~h}$ of hospital admission or (ii) within $48 \mathrm{~h}$ of hospital discharge [15].

Important changes in healthcare delivery over the last few years have seen some usually inpatient procedures now more often than not performed on an outpatient basis [18]. Patients transition freely within sometimes loosely defined levels of the health care system, for example between long-term care or rehabilitation services, to acute-care centres $[19,20]$. This study only considered hospital-acquired UTIs as opposed to a wider definition of healthcare associated UTIs, to avoid this confusion.

\section{Study selection}

The titles and abstracts of all papers identified in the electronic databases were examined and assessed for relevance and appropriateness to the principal objective of the systematic review. Irrelevant studies were excluded. Full texts of the potentially relevant papers were printed and carefully assessed against the systematic review inclusion and exclusion criteria. Those not meeting the criteria were excluded. The remaining papers deemed to have data relevant to the systematic review and meta-analysis were assessed for quality and risk of bias.

The study selection process and other stages of the review were performed by the lead author (OF). At each stage, $10 \%$ of papers identified were also screened against the study criteria independently by other authors (AG, GM and BM). Discrepancies in either the application of inclusion or exclusion of papers, quality assessment or on data extraction were discussed among all authors to make the final decision.

\section{Data extraction process}

Data were extracted by one author (OF) and $10 \%$ of papers eligible for data extraction were independently extracted by another author (AG). Data extraction was compared between AG and OF demonstrating $100 \%$ agreement for all items except the study design. This variable was therefore assessed by all authors. Where there was missing information on the study design of papers to be included in the meta-analysis, attempts were made to contact the authors. When there was no response, consensus on the study design was reached by all authors. Agreement between authors was assessed using Kappa coefficient. The agreement between all authors in deciding on the study design was $71 \%$ (Kappa $(95 \%$ CI $)=0.429(0.154-0.703), P$ Value $=0.003)$. Papers for which no agreement could be reached on the design, based on insufficient information, were assigned as nonclassifiable. Any other missing information in the included papers was recorded as 'not stated'.
The first author, year of study, country of study, study setting, age and sex distribution, co-morbidities, sample size, study design, study aim, antimicrobial susceptibility testing method, ciprofloxacin resistance rate, risk factors for ciprofloxacin resistance (i.e. previous antibiotic use) and mortality data (if reported) were extracted. Where the ciprofloxacin resistance rate was not available, the susceptibility rate was used to determine resistance.

\section{Risk of bias in individual studies}

Quality and risk of bias of the final papers included in the review was conducted using a modified version of the Newcastle-Ottawa Scale (NOS) which is a risk of bias assessment tool for observational studies recommended by the Cochrane Collaboration [21, 22]. Content validity and inter-rater reliability of this tool have been established [22]. Studies were rated by assigning a judgment of 'Low risk' of bias, 'High risk' of bias, or 'Unclear risk' of bias according to published criteria [21].

\section{Statistical analysis}

Pooled ciprofloxacin resistance proportions (with $95 \%$ confidence intervals) in patients with $E$. coli UTI were separately calculated and compared between hospital and community settings using a random-effects metaanalysis model based on DerSimonian and Laird method $[23,24]$. This method incorporates an estimate of the between-study variation into both the study weights and the standard error of the estimate of the common effect. The precision of an estimate from each included study was represented by the inverse of the variance of the outcome pooled across all studies. If the value of the pooled prevalence was within the $95 \% \mathrm{CI}$, then the effect size was statistically significant at the $5 \%$ level $(P<$ $0.05)$. The heterogeneity among studies was assessed by using the $\mathrm{I}^{2}$ statistic with a $P$ value of $<0.05$ considered statistically significant, and $\mathrm{I}^{2}$ values below $25 \%$ indicating low heterogeneity, 25-75\% moderate heterogeneity and over $75 \%$ high heterogeneity [25]. Subgroup analyses were done by risk of bias, study duration, age group, UTI symptoms, world region and economy of country (categorised as developed and developing using the World Bank classification [26]). A meta-regression analysis was used to determine the effect of measured covariates on the observed heterogeneity in resistance estimates across studies [23]. Assessment of publication bias was estimated using funnel plots. Further analysis was undertaken to examine pooled ciprofloxacin resistance over time using the median study year. For studies occurring over 2 years, the first year was used; for studies occurring over 4 years, the 2nd year was used; for those over 6 years, the 3rd year was used. The nonparametric Spearman's rho correlation coefficient was 
calculated to determine significance in resistance trend over time. Statistical analyses were undertaken using Stata statistical softwareversion 13 [27].

\section{Results}

Study selection

Electronic database searches identified 15,062 potential studies and 31 additional studies were identified through hand searching. After 11,397 duplicates were removed, 3696 articles remained for title and abstract screening. We assessed 1134 as potentially eligible and retrieved the full text of these articles. After applying inclusion and exclusion criteria, 53 papers $(5 \%)$ were deemed to have data relevant to the systematic review and metaanalysis. These 53 papers consisted of 54 studies comprising three hospital-acquired E. coli UTI studies and 51 community-acquired E. coli UTI studies. There was one paper that compared resistance in both hospital and community settings hence reported as two studies [15]. The PRISMA flow chart describing the papers identified from the search strategy and reasons for exclusion is shown in Fig. 1.

\section{Study characteristics}

Geographically, 53 of the 54 studies were carried out in Asia (28 \%; $n=15)$, Europe ( $24 \% ; n=13)$, Middle East $(15 \% ; n=8)$, Africa (13\%; $n=7)$, North America (11\%; $n=6)$ and South America $(7 \% ; n=4)$. The remaining study was conducted in multiple countries [28]. There were 17 (31\%) studies conducted in developed countries and $36(67 \%)$ in developing countries. The majority of the studies $(80 \%)$ followed a cross sectional design. The duration of studies ranged from 2 months to 84 months (median $=15.5 ; \mathrm{IQR}=12.0-30.0)$. The mean age and sex proportion of patients with an $E$. coli UTI were stated in $13 \%(n=7)$ and $44 \%(n=24)$ of studies respectively. Most study populations included patients of both sexes although $19 \%(n=10)$ included only women. Antimicrobial susceptibility testing and interpretation was performed using the disk diffusion method (74\%) and Clinical and Laboratory Standards Institute (CLSI) criteria (83 \%) respectively in most studies. Table 1 provides further details on the description of the included studies.

\section{Pooled ciprofloxacin resistance}

Figures 2 and 3 show the forest plots of studies reporting on ciprofloxacin resistance in community acquired E. coli UTI by region and economy, respectively. Figure 4 shows the forest plot of studies reporting on ciprofloxacin resistance in hospital acquired E. coli UTI. Compared with the community-setting, resistance to ciprofloxacin in $E$ coli UTIs was significantly higher in the hospital-setting $(P<0.001)$. Overall, the pooled rate for ciprofloxacin resistance in patients with community-acquired $E$. coli UTIs was 0.27 (95 \% CI: 0.240-0.310), compared with 0.38 (95 \% CI: 0.360-0.410) in the hospital setting. There was substantial heterogeneity among the community-setting studies $\left(I^{2}=98.8 \%, P<0.0001\right)$, but very little in the hospital ones $\left(I^{2}=<0.010 \%, P=0.641\right)$. Further analysis of studies reporting on community-acquired $E$. coli UTI by region (Fig. 3) showed that Asia had the highest pooled resistance. Analysis by economy based on the World Bank classification (Fig. 4) showed a higher pooled resistance in developing countries.

\section{Resistance over time in community-acquired UTI studies}

Figure 5 shows the scatter plot of ciprofloxacin resistance in 47 studies reporting on community-acquired UTI using the median study year for each study. Four studies did not provide data on the year(s) the study was conducted and were excluded from this analysis [29-32]. The results of the Spearman's rho correlation test showed a statistically significant rise in resistance over time $\left(n=47, r_{s}=\right.$ $0.431, P=0.003)$. Similar findings were observed for developing countries. There was no significant rise in resistance over time in developed countries.

\section{Subgroup analyses}

Sub-group analysis was conducted within each major setting. For community-acquired UTI studies (Table 2), there was a significant difference in the pooled resistance within each subgroup examined (risk of bias, study duration, economy, region, age group and UTI symptoms). The subgroup analyses results for studies reporting on hospital-acquired E. coli UTI (see Additional file 2) showed no difference in the pooled resistance within the subgroups examined (region, economy and UTI symptoms). When both settings were compared (see Additional file 3), there were significant differences noted for risk of bias (high), study duration ( $>12$ months), economy (developed), region (Americas), age group (adults and children) and UTI symptoms $(P<0.001)$. There were no data available on mortality for comparison between settings.

\section{Meta-regression analyses}

Random effects meta-regression analyses of studies reporting on community-acquired $E$. coli UTI showed that country's economy $(P=0.008)$, Asia as a region $(P=$ $0.002)$, high risk of bias $(P=0.003)$, year of study $(P=$ 0.020 ) and studies using only children as the study population $(P=0.030)$ were the study factors significantly accounting for the observed heterogeneity, responsible for $61 \%$ of the between study variance (Adjusted $\mathrm{R}^{2}$ ) in ciprofloxacin resistance.

\section{Risk of bias}

When studies were assessed for risk of bias using the Newcastle-Ottawa scale, $30 \%(n=16)$ were assessed as 


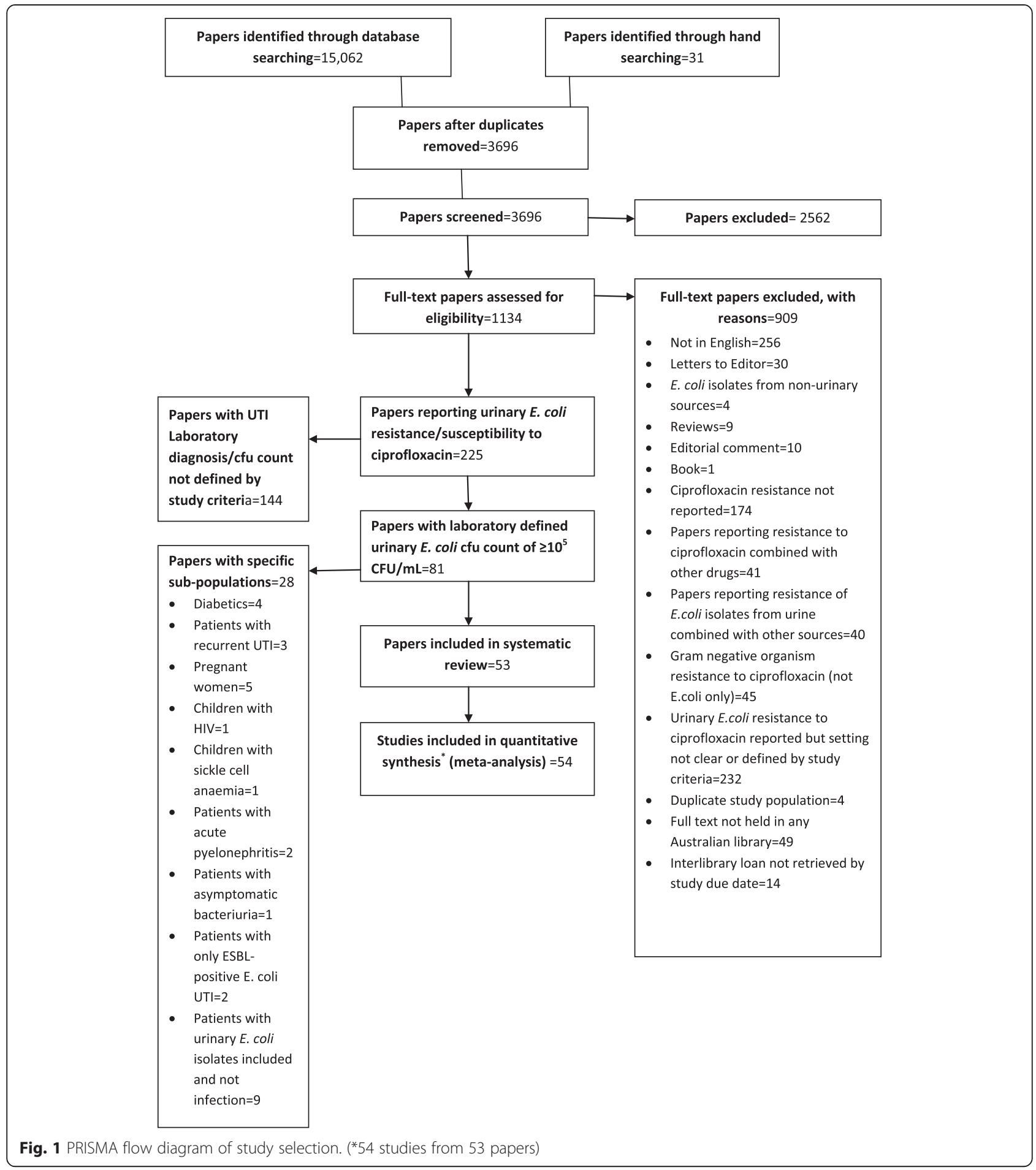

having a low risk of bias; $22 \%(n=12)$ unclear risk of bias and $48 \%(n=26)$ were deemed to have a high risk of bias. Further analysis of the 16 low risk studies only was consistent with findings reported from the analysis of all studies. An increasing resistance trend over time was also observed, however this increase did not reach statistical significance because of reduced statistical power.

\section{Discussion}

The findings of this systematic review and meta-analysis highlight the higher ciprofloxacin resistance in hospitalacquired E.coli UTI when compared to communityacquired UTI. There is also substantial evidence that ciprofloxacin resistance in community-acquired E. coli UTI has been increasing in recent years. Resistance was 
Table 1 Description of studies included in meta-analysis

\begin{tabular}{|c|c|c|c|c|c|c|c|c|c|c|}
\hline Study author & Country & Design $^{a}$ & Setting & Risk of bias & $\begin{array}{l}\text { Study duration } \\
\text { (months) }\end{array}$ & $\begin{array}{l}\text { Number of } \\
\text { positive E. coli } \\
\text { UTI samples }\end{array}$ & $\begin{array}{l}\text { Number of } \\
\text { ciprofloxacin } \\
\text { resistant } E \text {. coli }\end{array}$ & $\begin{array}{l}\text { Proportion resistant } \\
(95 \% \mathrm{Cl})\end{array}$ & Standard error & Weight $^{d}(\%)$ \\
\hline Ahmad, 2012 & India & Cross sectional & Community & Unclear & 24 & 318 & 48 & $0.15(0.11,0.19)$ & 0.02 & 2.09 \\
\hline Akoachere et al., 2012 & Cameroon & Cross sectional & Community & Low & 12 & 43 & 11 & $0.26(0.13,0.39)$ & 0.07 & 1.61 \\
\hline Akram et al., 2007 & India & Cross sectional & Community & High & 12 & 61 & 42 & $0.69(0.57,0.80)$ & 0.06 & 1.70 \\
\hline AlSweih et al., 2005 & Kuwait & Cross sectional & Community & High & 12 & 1535 & 81 & $0.05(0.04,0.06)$ & 0.01 & 2.15 \\
\hline Al-Tawfiq et al., 2009 & Saudi Arabia & Cohort & Community & High & 12 & 2281 & 592 & $0.26(0.24,0.28)$ & 0.01 & 2.14 \\
\hline Ansbach et al., 2013 & USA & Cross sectional & Community & High & 7 & 98 & 2 & $0.02(-0.01,0.05)$ & 0.01 & 2.12 \\
\hline Arabi et al., 2013 & Iran & Cross sectional & Community & Low & 33 & 103 & 23 & $0.22(0.14,0.30)$ & 0.04 & 1.91 \\
\hline Araujo et al., 2011 & Brazil & Cross sectional & Community & Unclear & 24 & 391 & 36 & $0.09(0.06,0.12)$ & 0.01 & 2.12 \\
\hline Arslan et al., 2005 & Turkey & Cross sectional & Community & Low & 5 & 514 & 135 & $0.26(0.22,0.30)$ & 0.02 & 2.09 \\
\hline Astal, 2005 & Palestine & Cross sectional & Community & High & 6 & 252 & 30 & $0.12(0.08,0.16)$ & 0.02 & 2.09 \\
\hline Azap et al., 2010 & Turkey & Cohort & Community & Unclear & 12 & 464 & 139 & $0.30(0.26,0.34)$ & 0.02 & 2.08 \\
\hline Bahadin et al., 2011 & Singapore & Cross sectional & Community & Unclear & 12 & 90 & 22 & $0.24(0.16,0.33)$ & 0.05 & 1.86 \\
\hline Biswas et al., 2006 & India & Cross sectional & Community & High & 36 & 354 & 124 & $0.35(0.30,0.40)$ & 0.03 & 2.05 \\
\hline Bouchillon et al., 2013 & USA & Cross sectional & Community & High & 24 & 723 & 234 & $0.32(0.29,0.36)$ & 0.02 & 2.10 \\
\hline Bouchillon et al., 2013 & USA & Cross sectional & Hospital & High & 24 & 253 & 103 & $0.41(0.35,0.47)$ & 0.03 & 11.83 \\
\hline Dash et al., 2013 & India & Cross sectional & Community & Low & 30 & 397 & 212 & $0.53(0.48,0.58)$ & 0.03 & 2.05 \\
\hline Dimitrov et al., 2004 & Kuwait & Cross sectional & Community & High & 84 & 780 & 92 & $0.12(0.10,0.14)$ & 0.01 & 2.13 \\
\hline Farshad et al., 2011 & Iran & Cross sectional & Community & Low & 12 & 90 & 8 & $0.09(0.03,0.15)$ & 0.03 & 2.01 \\
\hline Ghadiri et al., 2012 & Iran & Cross sectional & Hospital & High & 24 & 200 & 80 & $0.40(0.33,0.47)$ & 0.03 & 9.41 \\
\hline Gobernado et al., 2007 & Spain & Cross sectional & Community & Low & 12 & 2292 & 418 & $0.18(0.17,0.20)$ & 0.01 & 2.14 \\
\hline Ho et al., 2010 & Hong Kong & Cross sectional & Community & Low & 24 & 271 & 35 & $0.13(0.09,0.17)$ & 0.02 & 2.09 \\
\hline Hoban et al., 2011 & Multiple countries & Cross sectional & Hospital & High & 24 & 1643 & 624 & $0.38(0.36,0.40)$ & 0.01 & 78.76 \\
\hline Ismaili et al., 2011 & Belgium & Cohort & Community & High & 24 & 189 & 5 & $0.03(0.00,0.05)$ & 0.01 & 2.13 \\
\hline Kashef et al., 2010 & Iran & Cross sectional & Community & High & 30 & 578 & 180 & $0.31(0.27,0.35)$ & 0.02 & 2.09 \\
\hline Kiffer et al., 2007 & Brazil & Cross sectional & Community & Unclear & 48 & 22679 & 2699 & $0.12(0.11,0.12)$ & 0.002 & 2.15 \\
\hline Killgore et al., 2004 & USA & Case-control & Community & Low & 12 & 120 & 40 & $0.33(0.25,0.42)$ & 0.04 & 1.89 \\
\hline Kimando et al., 2010 & Kenya & Cross sectional & Community & Unclear & 6 & 92 & 6 & $0.07(0.01,0.12)$ & 0.03 & 2.05 \\
\hline Kothari et al., 2008 & India & Cross sectional & Community & High & 6 & 361 & 260 & $0.72(0.67,0.77)$ & 0.02 & 2.06 \\
\hline Kurutepe et al., 2005 & Turkey & NC & Community & High & 72 & 880 & 174 & $0.20(0.17,0.22)$ & 0.01 & 2.12 \\
\hline Lau et al., 2004 & Taiwan & Cross sectional & Community & Unclear & 13 & 80 & 14 & $0.17(0.09,0.26)$ & 0.04 & 1.89 \\
\hline Ljuca et al., 2010 & Bosnia \& Herzegovina & Cross sectional & Community & High & 36 & 43 & 4 & $0.09(0.01,0.18)$ & 0.04 & 1.87 \\
\hline
\end{tabular}


Table 1 Description of studies included in meta-analysis (Continued)

\begin{tabular}{|c|c|c|c|c|c|c|c|c|c|c|}
\hline Longhi et al., 2012 & Italy & $\mathrm{NC}$ & Community & Low & 6 & 154 & 36 & $0.23(0.17,0.30)$ & 0.03 & 1.98 \\
\hline Martinez et al., 2012 & Colombia & Cross sectional & Community & High & 2 & 102 & 39 & $0.38(0.29,0.48)$ & 0.05 & 1.83 \\
\hline Miragliotta et al., 2008 & Italy & Cohort & Community & Low & 60 & 2589 & 422 & $0.16(0.15,0.18)$ & 0.01 & 2.14 \\
\hline Molina-Lopez et al., 2011 & México & Cross sectional & Community & High & 48 & 119 & 65 & $0.55(0.46,0.64)$ & 0.05 & 1.86 \\
\hline Moreira et al., 2006 & Brazil & Cross sectional & Community & Unclear & 15 & 544 & 65 & $0.12(0.09,0.15)$ & 0.01 & 2.12 \\
\hline Murugan et al., 2012 & India & Cohort & Community & High & 12 & 204 & 144 & $0.71(0.64,0.77)$ & 0.03 & 2.00 \\
\hline Muvunyi et al., 2011 & Rwanda & Cross sectional & Community & Low & 6 & 72 & 23 & $0.32(0.21,0.43)$ & 0.05 & 1.75 \\
\hline Mwaka et al., 2011 & Uganda & Cross sectional & Community & High & NS & 27 & 9 & $0.33(0.16,0.51)$ & 0.09 & 1.32 \\
\hline Ni Chulain et al., 2005 & Ireland & Cross sectional & Community & High & 5 & 723 & 18 & $0.02(0.01,0.04)$ & 0.01 & 2.15 \\
\hline Olson et al., 2012 & USA & Cross sectional & Community & Unclear & 16 & 95 & 4 & $0.04(0.00,0.08)$ & 0.02 & 2.08 \\
\hline Otajevwo, 2013 & Nigeria & Cross sectional & Community & High & 6 & 5 & 4 & $0.80(0.45,1.15)$ & 0.18 & 0.63 \\
\hline Prakash et al., 2013 & India & Cross sectional & Community & Low & NS & 23 & 16 & $0.70(0.51,0.88)$ & 0.10 & 1.26 \\
\hline Randrianirina et al., 2007 & Madagascar & Cross sectional & Community & Low & 28 & 607 & 100 & $0.16(0.14,0.19)$ & 0.02 & 2.12 \\
\hline Rani et al., 2011 & India & Cross sectional & Community & Unclear & 6 & 208 & 151 & $0.73(0.67,0.79)$ & 0.03 & 2.01 \\
\hline Shaifali et al., 2012 & India & Cross sectional & Community & Unclear & 12 & 46 & 28 & $0.61(0.47,0.75)$ & 0.07 & 1.54 \\
\hline Shariff et al., 2013 & India & Cross sectional & Community & High & 18 & 491 & 160 & $0.33(0.28,0.37)$ & 0.02 & 2.08 \\
\hline Sire et al., 2007 & Senegal & Cross sectional & Community & Low & 33 & 1010 & 157 & $0.16(0.13,0.18)$ & 0.01 & 2.13 \\
\hline Sood et al., 2012 & India & NC & Community & High & 30 & 214 & 160 & $0.75(0.69,0.81)$ & 0.03 & 2.02 \\
\hline Stratchounski et al., 2006 & Russia & NC & Community & Low & 48 & 423 & 18 & $0.04(0.02,0.06)$ & 0.01 & 2.14 \\
\hline Vellinga et al., 2012 & Ireland & Case-control & Community & Low & 9 & 633 & 78 & $0.12(0.10,0.15)$ & 0.01 & 2.12 \\
\hline Wang et al., 2014 & China & Cross sectional & Community & High & 8 & 129 & 91 & $0.71(0.63,0.78)$ & 0.04 & 1.92 \\
\hline Yildirim et al., 2010 & Turkey & Cross sectional & Community & Unclear & 24 & 450 & 85 & $0.19(0.15,0.23)$ & 0.02 & 2.10 \\
\hline Yolbas et al., 2013 & Turkey & Cross sectional & Community & High & 12 & 113 & 24 & $0.21(0.14,0.29)$ & 0.04 & 1.93 \\
\hline
\end{tabular}

${ }^{\text {aNon-classifiable design }}$

${ }^{\mathrm{b}}$ Not stated

'Study denominator

'Weights are from random effects analysis using DerSimonian-Laird model 


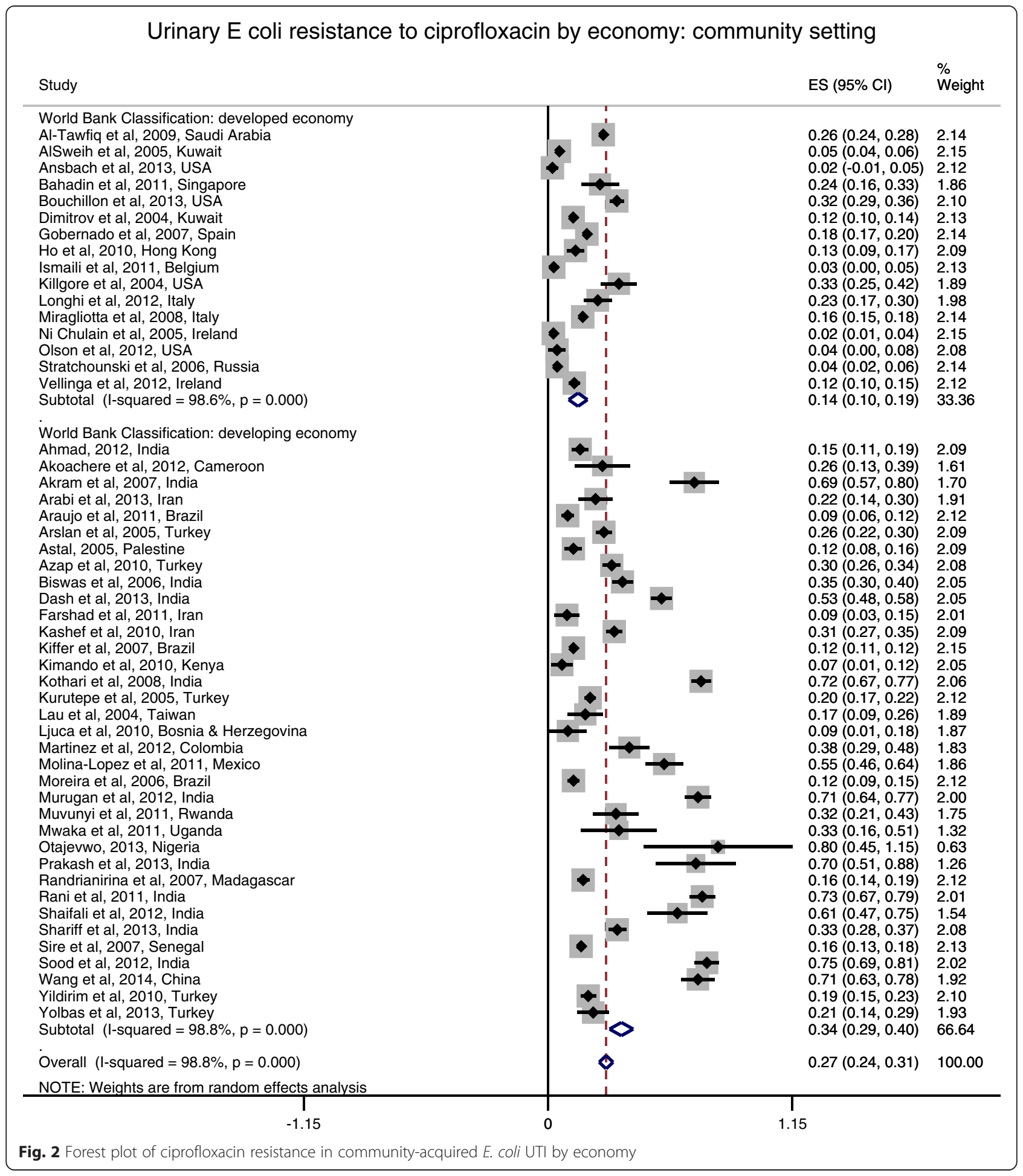

also found to be significantly higher in developing countries reporting on $E$. coli UTI in community settings.

Antimicrobial resistance has been described as an international hazard to public health threatening the successful prevention and treatment of bacterial, viral, parasitic and fungal infections [3, 33]. As such, research into its prevention and reduction is very important. Our estimated pooled ciprofloxacin resistance of 27 and $38 \%$ in community- and hospital-acquired E. coli UTI respectively could not be compared to any other systematic review findings because, to our knowledge, this is the first systematic review and meta-analysis comparing ciprofloxacin 


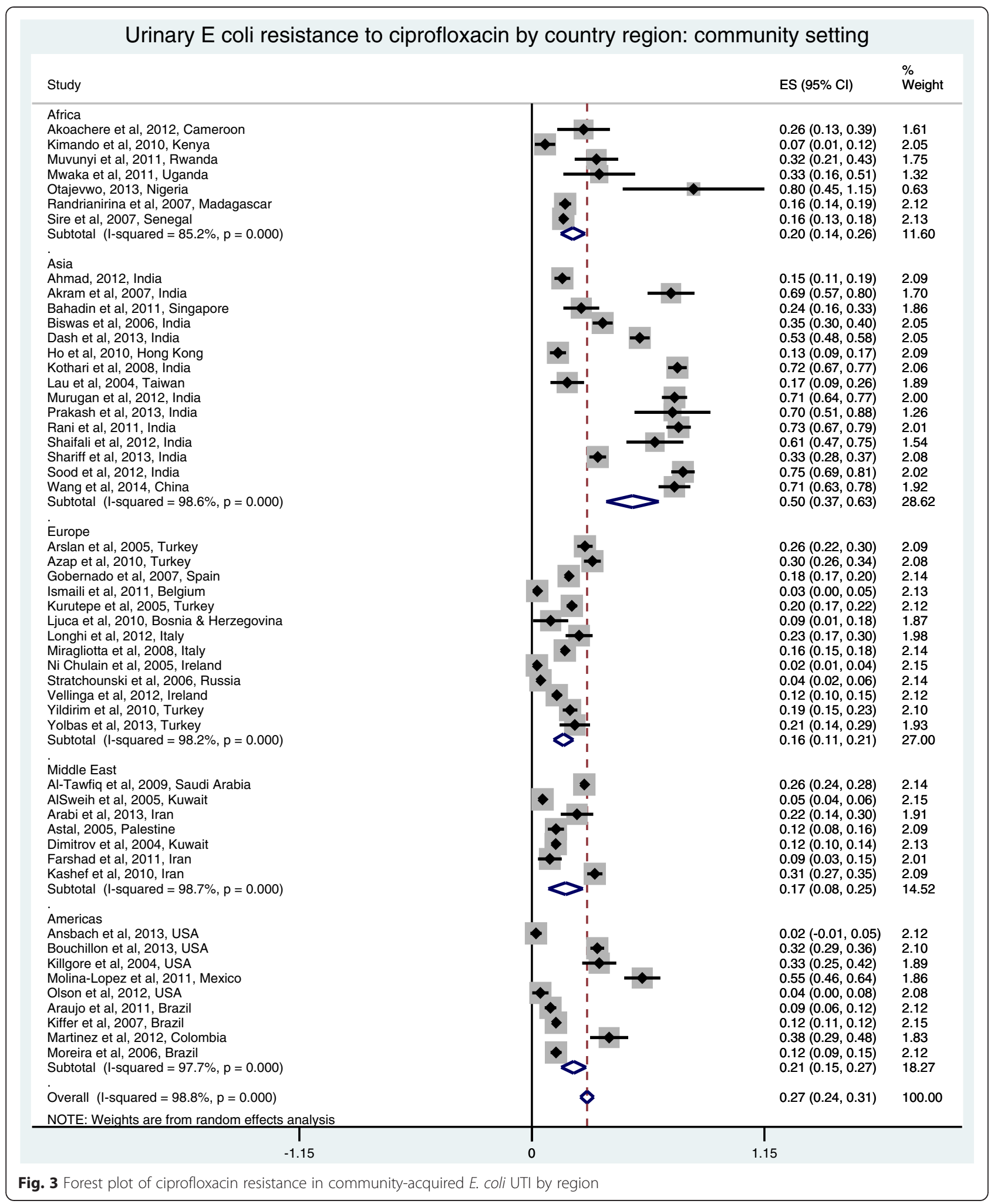

resistance in community- and hospital-acquired E. coli UTI. However, national data from five WHO regions show at least $50 \%$ resistance to fluoroquinolones (ciprofloxacin, norfloxacin or ofloxacin) in E. coli [3]. Data on E. coli in the WHO report are from various settings and sources (including blood and urine) hence cannot be directly compared with the results from our systematic review. Another recent review on global fluoroquinolone resistance 
Urinary E coli resistance to ciprofloxacin: hospital setting

Study

ES $(95 \% \mathrm{Cl})$

Weight

Bouchillon et al, 2013, USA

Ghadiri et al, 2012, Iran

Hoban et al, 2011, multiple countries

Overall $(I-$ squared $=0.0 \%, p=0.641)$

NOTE: Weights are from random effects analysis
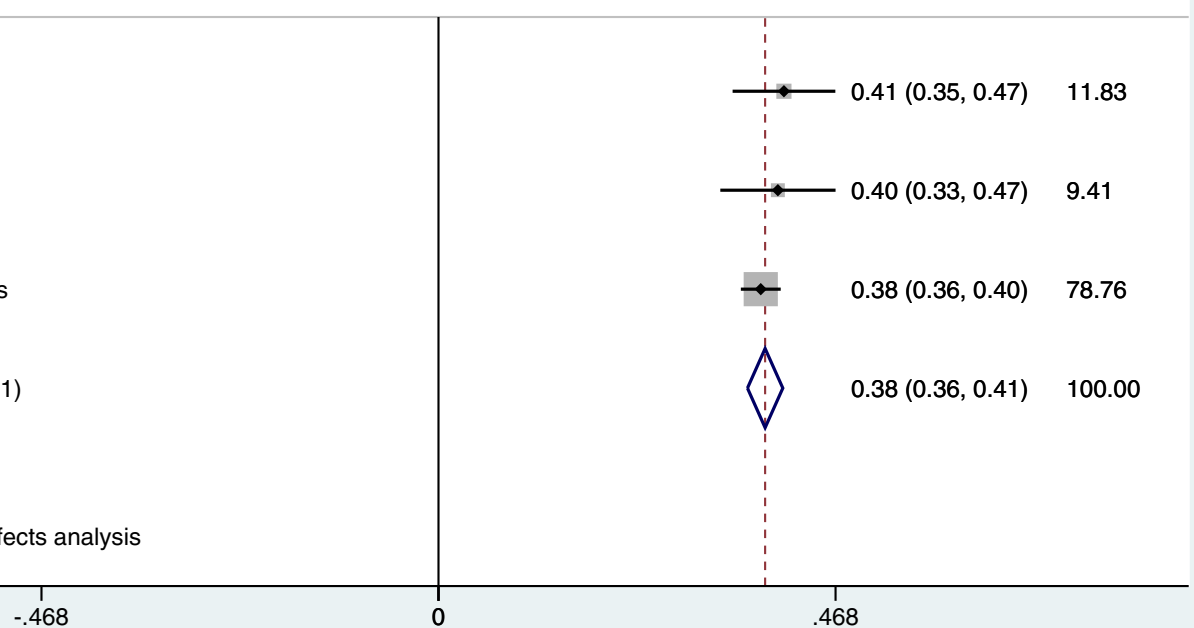

Fig. 4 Forest plot of ciprofloxacin resistance in hospital-acquired E. coli UTI

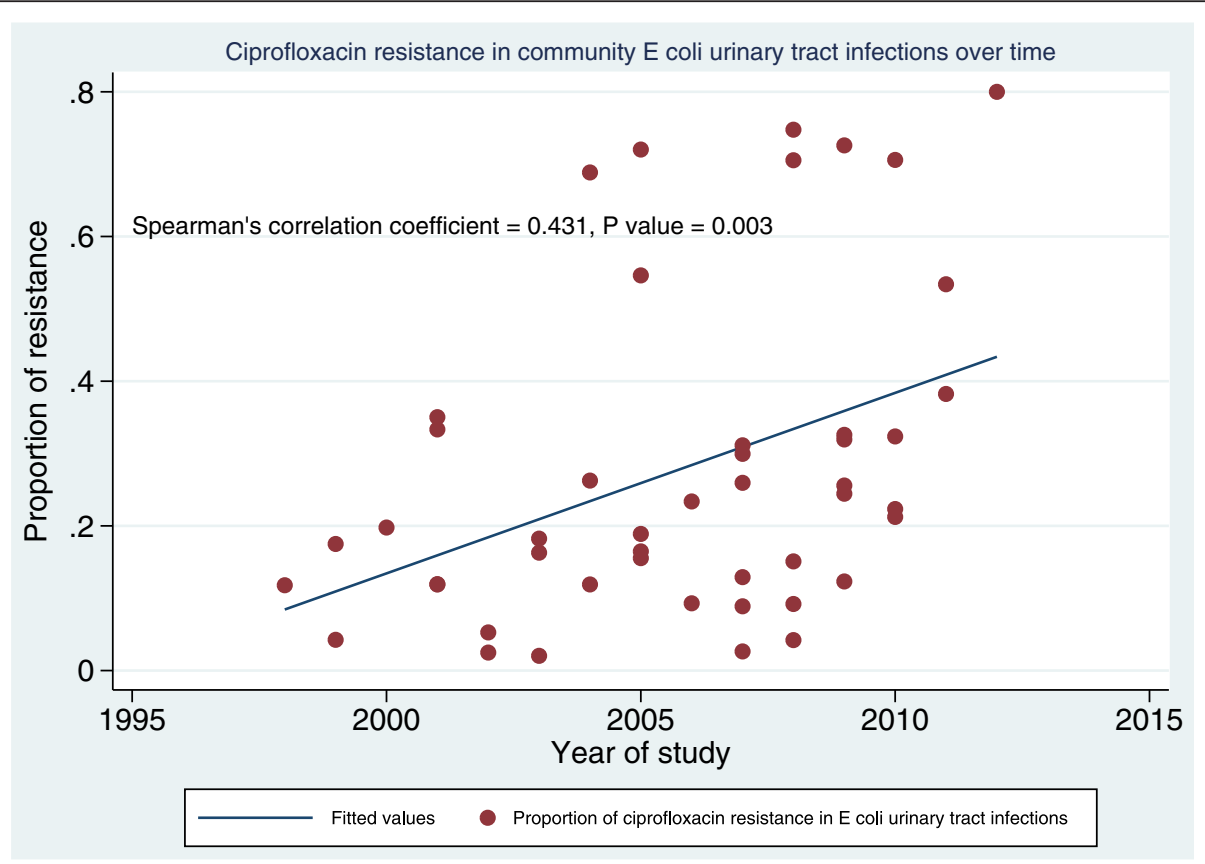

Fig. 5 Scatter plot of ciprofloxacin resistance in community-acquired UTI by year of study (1998-2012). N=47 (4 studies excluded due to missing information on year study was conducted) 
Table 2 Subgroup analyses of pooled ciprofloxacin resistance in community setting

\begin{tabular}{|c|c|c|c|}
\hline \multirow[t]{2}{*}{ Subgroup } & & Community Setting $N=51$ & $P$ value* \\
\hline & & \multicolumn{2}{|l|}{ Pooled resistance } \\
\hline \multirow[t]{2}{*}{ Risk of bias } & Low and unclear $n=28$ studies & 0.221 & $<0.0001$ \\
\hline & High $n=23$ studies & 0.337 & \\
\hline \multirow[t]{2}{*}{ Study duration ${ }^{a}$} & $\leq 12$ months $n=25$ studies & 0.323 & $<0.0001$ \\
\hline & $>12$ months $n=24$ studies & 0.219 & \\
\hline \multirow[t]{2}{*}{ Economy } & Developed $n=16$ studies & 0.141 & $<0.0001$ \\
\hline & Developing $n=35$ studies & 0.345 & \\
\hline \multirow[t]{2}{*}{ Region } & Africa, Asia and Middle East $n=29$ studies & 0.361 & $<0.0001$ \\
\hline & Europe, North and South American $=22$ studies & 0.174 & \\
\hline \multirow[t]{2}{*}{ Age group $^{a}$} & Adults and children ${ }^{b} n=24$ studies & 0.265 & $<0.0001$ \\
\hline & Adults onlyn $=19$ studies & 0.302 & \\
\hline \multirow[t]{2}{*}{ UTI symptoms } & Symptomatic and asymptomatic patients $n=11$ studies & 0.185 & $<0.0001$ \\
\hline & Symptomatic patients only $n=40$ studies & 0.295 & \\
\hline
\end{tabular}

$n=$ number of studies reporting on community acquired UTI

*Comparing pooled resistance for difference in subgroup in community setting

${ }^{\text {a }}$ Studies with missing information on this sub-analysis were not included

bStudies reporting resistance in adults and children or children only

epidemiology reported a range of 2 to $69 \%$ for fluoroquinolone resistance in uncomplicated communityacquired UTI and up to $98 \%$ in complicated cases, with fluoroquinolone resistance in healthcare associated UTIs ranging from 6 to $62 \%$ [34]. The findings from our systematic review are within the above reported ranges. However, the latter ranges were wide and the data were from a number of different Gram negative uropathogens and not specifically $E$. coli accounting for the higher rates. Available published data show relatively high rates of urinary E. coli resistance to ciprofloxacin [35-41] prompting the need for a renewed effort in the further prevention of spread of resistance to this antimicrobial agent.

We found that urinary E. coli resistance to ciprofloxacin was higher in the hospital compared to the community setting. Our finding is comparable to individual studies which have assessed urinary E.coli resistance to ciprofloxacin in both, hospital and community settings [31, 41-45]. However, often studies do not apply the criterion of $48 \mathrm{~h}$ post admission used in our systematic review for identifying hospital acquired UTI $[45,46]$. The Canadian national surveillance study (CANWARD), a large population-based study undertaken from 2007 to 2009, further confirms our finding of higher resistance in the hospital setting [47]. Inpatients had a significantly higher urinary E. coli resistance to ciprofloxacin. Similar findings were reported by Cullen et al. in Dublin [16]. This is not an unusual finding and may be attributed to the selective pressure resulting from antimicrobial use in hospital settings [47]. Patients in hospital, already acutely ill, become more at risk of developing a resistant infection because of potential immune deficiency and relative high exposure to antimicrobial agents [48]. Furthermore, hospitalized patients are more likely to be exposed to practices that result in cross infection or transmission of organisms. These and other risk factors enable the spread of resistance. This has significant implications for patient care as antimicrobial resistance may lead to treatment failure resulting in death.

The results of our systematic review showed a significant rise in resistance over time in the community setting. This finding is supported by a number of US-based studies investigating antimicrobial resistance trend in outpatients. A fivefold increase (from 3 to $17.1 \%$ ) in ciprofloxacin resistance was observed from 2000 to 2010 by Sanchez et al. [17] in comparison with other antibiotics investigated [49]. Our findings are also consistent with Blaettler et al. [6] who found that over a 10 year period (1997-2007), similar to the timeframe for our review, resistance increased significantly for ciprofloxacin from 1.8 to $15.9 \%$ in Switzerland. This increase coincided with a rise in ciprofloxacin use in Switzerland [6]. These findings suggest that with increase in the use of fluoroquinolones generally over time, resistance ciprofloxacin is likely to further increase. It is now known that antimicrobial overuse or misuse is a risk factor for the development of AMR [50]. The specific effect of ciprofloxacin use on the development of its resistance in UTI pathogens is also clearly documented. A recent Irish study involving 72 general practices found higher ciprofloxacin resistance levels $(5.5 \%)$ in practices with 10 prescriptions per month compared with resistance levels of $3 \%$ in practices with one prescription per month [51]. Wide spread use of this agent may have thus resulted in a rise in ciprofloxacin resistance. In the Netherlands and United States, an association has also been shown between high 
fluoroquinolone prescriptions and a rise in bacterial resistance [52, 53]. Furthermore, changes in antimicrobial prescribing practices have been shown to precede changes in resistance rates. A study by Gottesman et al. [54] in Israel found a significant decrease in E. coli resistance to ciprofloxacin following a nationwide restriction on ciprofloxacin use. Resistance decreased from $12 \%$ in the pre-intervention period to $9 \%$ in the intervention period. Our results pose a strong argument for the development of more stringent criteria limiting ciprofloxacin use. In addition, other strategies such as adequate surveillance and monitoring, reinforcement of existing infection prevention and control measures as well as new technological advancement will help reduce the widespread problem of antimicrobial resistance [55-57] but these aspects are not within the scope of this paper.

Our finding of a significant rise in resistance over time also has implications for the development of treatment guidelines. The national recommendations for first-choice empiric antibiotic treatment of UTIs vary considerably [5]. In countries like Spain, Taiwan and Turkey, the treatment choice for uncomplicated UTIs are fluoroquinolones $[5,58$, 59]. In 2000, fluoroquinolones were prescribed for treatment of uncomplicated UTIs in Switzerland in $64 \%$ of cases [60]. There is concern that resistance to ciprofloxacin resulting from its first-line use may be associated with an increase in multidrug resistance [61]. The most recent IDSA guidelines [9] advise using nitrofurantoin, trimethoprimsulphamethoxazole, fosfomycin or pivmecillinam for first-line treatment of acute uncomplicated cystitis. Fluoroquinolones should be reserved for important uses other than acute cystitis or used as an alternative only when these recommended agents cannot be used [9]. We recommend that ciprofloxacin should not be used as a first line treatment option for UTIs as continuous increases in resistance to ciprofloxacin further weaken the effectiveness of this drug.

Additional findings from the meta-analysis showed that resistance was significantly higher in developing countries compared to developed countries. A major factor accounting for this difference is the use of over the counter or non-prescription antibiotics which occur commonly in developing countries $[62,63]$. Although this review did not directly consider antimicrobial resistance in relation to prescribing for the included studies, evidence shows that over the counter or non-prescription use results in unnecessary and excessive use of antibiotics. Some of the included studies in our review clearly state that there are no restrictions for over the counter prescribing of antimicrobials within their countries [29, 64-73]. A recent systematic review investigating global non-prescription antimicrobial use found that resistance was common in communities with frequent non-prescription antimicrobial use [74]. Non-prescription use was highest in Africa, Asia and Middle East at 100, 58 and $39 \%$ respectively [74]. In our review, further analyses by region showed that Asia had the highest pooled resistance to ciprofloxacin with a significantly higher resistance in Africa, Asia and Middle East combined compared with Europe and the Americas. Our finding is supported by a recent paper by Dalhoff [75] reporting that fluoroquinolone resistance was highest in the Asia-Pacific region and moderate to low in Europe and North America. Furthermore, there is evidence to show that countries that have developed control policies to regulate non-prescription use have seen a decrease in antimicrobial use and resistance rates [74]. Based on our findings, we therefore emphasize the need for the development of policies restricting over the counter antimicrobial use in countries that do not have such policies thereby contributing to the prevention of patient morbidity and mortality associated with resistant infections. It is noteworthy to mention that another important factor contributing to antimicrobial resistance is the use of antibiotics in livestock for growth promotion [76]. Extensive antimicrobial use in food animal production has been associated with antimicrobial resistance globally [76]. This has considerable implications for human health with the need to protect the efficacy of these antimicrobials to ensure their effectiveness for the treatment of humans.

A large variation in ciprofloxacin resistance was found in studies reporting on community-acquired UTI. This variation highlights the significance of local resistance monitoring to guide the development of local antibiotic guidelines. The random effects meta-regression model confirmed that a number of factors significantly accounted for the variations in ciprofloxacin resistance. These include economy (developed and developing), Asia as a region, year of study, studies including only children and studies with a high risk of bias. The first three factors have been discussed in detail in the preceding paragraphs. We found that resistance was lower in studies involving only children. This finding is in line with a number of studies which have compared resistance in adults and children showing significantly higher ciprofloxacin resistance in adults compared to children [77, 78]. Increased age has also been shown to be significantly associated with ciprofloxacin resistance [6, 47]. Given that children are less exposed to antimicrobials with limited ciprofloxacin use in the paediatric age group, this finding is expected [77-79]. Although the importance of intrafamilial cross-infection of resistant pathogens is yet to be confirmed, it has been suggested that fluoroquinolone resistance may to some extent be dependent on cross-infection with transfer from adults to children [78]. Given this assumption, it is necessary to also monitor resistance levels in children to prevent further resistance development in this vulnerable age group. Other likely causes of higher resistance in adults may be the greater likelihood of comorbidities with more 
frequent contact with healthcare settings [47]. The last factor found to account for heterogeneity between studies was high risk of bias. Most of the studies included in the review were found to have a high risk of bias as assessed using the NOS scale. These studies lacked methodological rigour including absence of the inclusion of possible confounding factors (such as age, sex and previous use of an antimicrobial) in the design and analysis of the studies. The poor reporting of observational studies poses limitations for conducting meta-analysis of these studies. Better presentation of definitions would enable inclusion in systematic reviews of some categories that had to be excluded in this review. Observational studies are more prone to confounding bias [80] further emphasizing the need for adherence to reporting guidelines such as such as that based on the Strengthening the Reporting of Observational Studies in Epidemiology (STROBE) Statement [81] to ensure clear and comprehensive reporting prior to publication acceptance. The poor quality of many studies initially retrieved for this review resulted in a large number being excluded. Therefore the information provided in this systematic review and meta-analysis of 54 observational studies may not sufficiently address ciprofloxacin resistance globally but may provide satisfactory evidence to inform future interventions.

In addition, this systematic review highlights the weaknesses in the quality of antimicrobial resistance data that are being collected in various regions. These weaknesses have implications for development of effective surveillance systems to monitor resistance globally and strategies to prevent further resistance development. The need for the implementation of national and global surveillance systems to detect and continuously monitor AMR cannot be overemphasized. These systems would enable prospective studies to be conducted and would play a major role in curtailing the widespread effect of antimicrobial resistance and help healthcare providers in deciding on the most appropriate empirical therapy for UTI to ensure proper management of patients. Governments need to put in place policies to restrict over the counter use and inappropriate prescribing of ciprofloxacin and other antimicrobials to prevent further development of resistance.

\section{Strengths and limitations}

There are a number of notable strengths to our review. To our knowledge, this is the first systematic review to compare the overall prevalence of ciprofloxacin resistance in community- and hospital-acquired E. coli UTI. We undertook a comprehensive literature search process to identify and screen articles against eligibility criteria. Given that generic versions of ciprofloxacin were first marketed at different times in various countries, our choice of 2004 as the start date was therefore made on the basis of changes in the epidemiology of antimicrobial resistant pathogens which had resulted in changes to treatment regimens. A further strength of this systematic review is the development of a peer reviewed, registered protocol prior to undertaking the review. For studies to be included in the review, they were restricted to those that used a standard laboratory UTI criterion of $\geq 10^{5} \mathrm{cfu} / \mathrm{mL}$ as recommended by the $\mathrm{CDC}$. Although applying the internationally recognised CDC criteria may definitely be considered a strength as it ensures the quality and uniformity of included studies, this criterion limited the number of hospital-acquired UTI studies included in our systematic review. Despite this, resistance was still found to be higher in the hospital setting compared to the community setting similar to published studies. While lower counts of uropathogens are relevant for acute episodes of uncomplicated cystitis, the use of different colony counts makes comparison of data between studies difficult. Including all urinary E.coli isolates was considered but not done because this existing surveillance criterion $\left(\geq 10^{5} \mathrm{cfu} / \mathrm{mL}\right.$ and $48 \mathrm{~h}$ cut off) is usually applied to defining infections not isolates. Also, including all isolates carries the risk of including duplicates. This approach poses some degree of ascertainment bias as our systematic review focuses on laboratory identified UTIs which may not only underestimate the total number of UTIs but also lead to selection of samples from complicated cases thereby overestimating resistance. Another limitation is the wide variation of resistance estimates between studies and the inclusion of studies having substantial clinical and methodological heterogeneity. Visual inspection of the funnel plot (Fig. 6) showed asymmetry suggesting evidence of publication bias, with studies reporting high resistance rates being more likely to be published posing a limitation to this review. Also, the quality and risk of bias of some of the studies included in the review were assessed as high. These limitations were addressed by undertaking a random effects meta-analysis with subsequent subgroup analyses and random effects metaregression to explain the sources of heterogeneity. For studies in which the design was not stated, the review authors faced difficulties in categorising such studies hence some of these studies were grouped as non-classifiable. These studies did not provide clear and explicit information on the methods used for conducting the studies. This emphasizes the need for implementation and adherence to clear reporting standards prior to publication of papers. Furthermore, in some included studies, adjustments were not made for important confounding factors relevant to antimicrobial resistance such as antibiotic use and patient demographics including age and sex. For this systematic review, studies on samples obtained from emergency department (ED) patients were classified as communityacquired samples. Included papers did not provide any information on whether some of these patients may have 


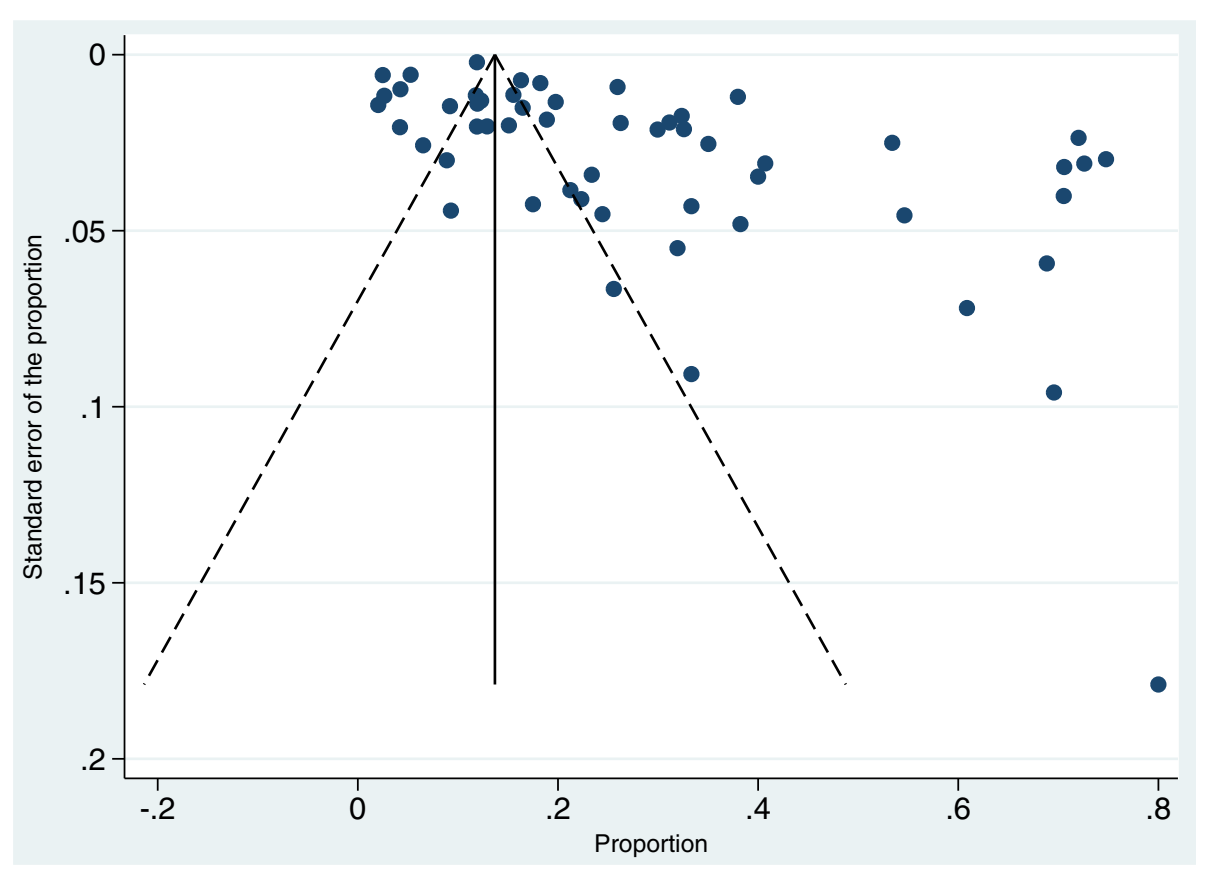

Fig. 6 Funnel plot of studies included in meta-analysis

returned from a recent hospitalisation and represented to the ED. Ideally, these should be considered as hospitalacquired infections as some of these patients may have been discharged in the previous $48 \mathrm{~h}$. For the purpose of this review and to overcome inherent variations in how individual studies have defined these patients, we classified all papers reporting on ED patients as communityacquired UTI studies. It was not possible to determine the potential effect of samples obtained from nursing home or residential aged care studies on the pooled resistance because this participant group did not meet the inclusion criteria for analysis. Furthermore, classification of this setting as hospital or community remains controversial. Finally, validity issues may have arisen from the use of different antimicrobial susceptibility test and interpretation methods with differing breakpoints which tend to change over the years. To date, there is still no worldwide consensus on the most suitable antimicrobial susceptibility testing method with the fact that various countries and even laboratories within the same country use different tests and interpretative criteria. Subgroup analysis for AST method was considered but not done because almost all studies used the disk diffusion method and CLSI criteria.

\section{Conclusions}

Ciprofloxacin resistance in E. coli UTI is increasing. The use of this antimicrobial agent as empirical therapy for UTI should be reconsidered and efforts should be made to limit its use to clinical conditions for which there are clear therapeutic indications. Policy restrictions on ciprofloxacin use need to be developed and enforced especially in developing countries that are yet to have such policies put in place. Further research is needed to describe ciprofloxacin resistance in hospital-acquired $E$. coli UTI using widely accepted definitions.

\section{Availability of Data and Materials}

Data supporting the findings of this systematic review are available upon request from the corresponding author.

\section{Additional files}

Additional file 1: Search strategy by database. Search of EMBASE, CINHAL, Scopus, PubMed, MEDLINE and COCHRANE. (PDF $58 \mathrm{~kb}$ )

Additional file 2: Subgroup analyses of pooled ciprofloxacin resistance in hospital setting. Results of subgroup analyses for studies reporting on hospital acquired E. coli UTI. (PDF $12 \mathrm{~kb}$ )

Additional file 3: Subgroup analyses of pooled ciprofloxacin resistance by setting. Results of subgroup analyses comparing the pooled resistance within each subgroup examined for both community and hospital settings. (PDF $36 \mathrm{~kb}$ )

\section{Competing interests}

The authors declare that they have no competing interests.

\section{Authors' contributions}

OF conceived the idea for this study and took primary responsibility for writing the protocol. AG, GM and BM contributed to the development of the idea and supervised the writing of the protocol. OF searched the literature and made the primary selection of eligible papers including data extraction. AG, GM and BM supervised and checked the study selection process, data extraction and data analysis plan. OF and GM analysed the data. All authors contributed to interpretation of the analysis. OF wrote the manuscript. AG, 
GM and BM critically reviewed and contributed to the manuscript. All authors have seen and approved the final version.

\section{Acknowledgements}

We would like to thank Associate Professor Elizabeth Mclnnes in reviewing the protocol and Ms Verena Schadewaldt for assisting with data retrieval.

\section{Funding}

There was no dedicated funding for this study. This study was carried out as part of a doctoral research program. OF is supported by an Australian Catholic University Postgraduate Award.

\section{Author details}

${ }^{1}$ Faculty of Health Sciences, Australian Catholic University, 223 Antill Street, Watson, Australian Capital Territory 2602, Australia. ${ }^{2}$ Faculty of Arts, Nursing and Theology, Avondale College of Higher Education, 185 Fox Valley Road, Wahroonga, New South Wales 2076, Australia. ${ }^{3}$ School of Allied Health, Faculty of Health Sciences, Australian Catholic University, 17 Young Street, Fitzroy, Victoria 3065, Australia.

Received: 15 July 2015 Accepted: 17 November 2015 Published online: 25 November 2015

\section{References}

1. Laupland K, Ross T, Pitout J, Church D, Gregson D. Community-onset urinary tract infections: a population-based assessment. Infection. 2007;35:150-3.

2. Gupta K, Hooton TM, Stamm WE. Increasing antimicrobial resistance and the management of uncomplicated community-acquired urinary tract infections. Ann Intern Med. 2001;135:41-50.

3. World Health Organisation. Antimicrobial resistance: global report on surveillance. 2014. http:/www.who.int/drugresistance/documents/surveillancereport/en/. Accessed 25 Oct 2014.

4. Schaeffer AJ. The expanding role of fluoroquinolones. Am J Med. 2002;113:45-54.

5. Mcquiston Haslund J, Rosborg Dinesen M, Sternhagen Nielsen AB, Llor C, Bjerrum L. Different recommendations for empiric first-choice antibiotic treatment of uncomplicated urinary tract infections in Europe. Scand J Prim Health. 2013;31:235-40

6. Blaettler L, Mertz D, Frei R, Elzi L, Widmer A, Battegay M, et al. Secular trend and risk factors for antimicrobial resistance in Escherichia coli isolates in Switzerland 1997-2007. Infection. 2009;37:534-9.

7. World Health Organisation Advisory Group on Integrated Surveillance of Antimicrobial Resistance. Critically Important Antimicrobials for Human Medicine-3rd Revision. 2012. http://apps.who.int/iris/bitstream/10665/77376/ 1/9789241504485_eng.pdf. Accessed 5 Dec 2014.

8. Redgrave LS, Sutton SB, Webber MA, Piddock LJV. Fluoroquinolone resistance: mechanisms, impact on bacteria, and role in evolutionary success. Trends Microbiol. 2014;22:438-45.

9. Gupta K, Hooton TM, Naber KG, Wullt B, Colgan R, Miller LG, et al. International clinical practice guidelines for the treatment of acute uncomplicated cystitis and pyelonephritis in women: a 2010 update by the Infectious Diseases Society of America and the European Society for Microbiology and Infectious Diseases. Clin Infect Dis. 2011;52:e103-e20.

10. Linhares I, Raposo T, Rodrigues A, Almeida A. Frequency and antimicrobial resistance patterns of bacteria implicated in community urinary tract infections: a 10-year surveillance study (2000-2009). BMC Infect Dis. 2013;13:19.

11. Ma KL, Wang CX. Analysis of the spectrum and antibiotic resistance of uropathogens in vitro: Results based on a retrospective study from a tertiary hospital. Am J Infect Control. 2013;41:601-6.

12. United States Interagency Task Force on Antimicrobial Resistance. A Public Health Action Plan to Combat Antimicrobial Resistance 2012 Update. 2012 http://www.cdc.gov/drugresistance/pdf/action-plan-2012.pdf. Accessed 5 Dec 2014.

13. Liberati A, Altman DG, Tetzlaff J, Mulrow C, Gøtzsche PC, loannidis JP, et al The PRISMA statement for reporting systematic reviews and meta-analyses of studies that evaluate health care interventions: explanation and elaboration. Ann Intern Med. 2009;151:W-65-94.

14. Centers for Disease Control and Prevention. Urinary Tract Infection (CatheterAssociated Urinary Tract Infection [CAUTI] and Non-Catheter-Associated Urinary Tract Infection [UTI]) and Other Urinary System Infection [USI]) Events. 2015. http://www.cdc.gov/nhsn/PDFs/pscManual/7pscCAUTlcurrent.pdf. Accessed 10 Feb 2015.
15. Bouchillon SK, Badal RE, Hoban DJ, Hawser SP. Antimicrobial Susceptibility of Inpatient Urinary Tract Isolates of Gram-Negative Bacilli in the United States: Results from the Study for Monitoring Antimicrobial Resistance Trends (SMART) Program: 2009-2011. Clin Ther. 2013;35:872-7.

16. Cullen IM, Manecksha RP, McCullagh E, Ahmad S, O'Kelly F, Flynn RJ, et al. The changing pattern of antimicrobial resistance within 42,033 Escherichia coli isolates from nosocomial, community and urology patient-specific urinary tract infections, Dublin, 1999-2009. BJUI. 2012;109:1198-206.

17. Sanchez GV, Master RN, Karlowsky JA, Bordon JM. In vitro antimicrobial resistance of urinary Escherichia coli isolates among US outpatients from 2000 to 2010. Antimicrob Agents Chemother. 2012;56:2181-3.

18. Horcajada JP, Shaw E, Padilla B, Pintado V, Calbo E, Benito N, et al. Healthcare-associated, community-acquired and hospital-acquired bacteraemic urinary tract infections in hospitalized patients: a prospective multicentre cohort study in the era of antimicrobial resistance. Clin Microbiol Infec. 2013;19:962-8.

19. Henderson KL, Muller-Pebody B, Johnson AP, Wade A, Sharland M, Gilbert R. Community-acquired, healthcare-associated and hospital-acquired bloodstream infection definitions in children: a systematic review demonstrating inconsistent criteria. J Hosp Infect. 2013;85:94-105.

20. Sydnor ER, Perl TM. Hospital epidemiology and infection control in acutecare settings. Clin Microbiol Rev. 2011;24:141-73.

21. Higgins J, Green S. Cochrane Handbook for Systematic Reviews of Interventions Version 5.1.0. The Cochrane Collaboration. 2011. http://www. cochrane-handbook.org. Accessed 10 Nov 2014.

22. Wells $\mathrm{G}$, Shea B, O'Connell D, Peterson J, Welch V, Losos M et al. The Newcastle-Ottawa Scale (NOS) for assessing the quality of nonrandomised studies in meta-analyses. 2014. http://www.ohri.ca/programs/_linical_ epidemiology/oxford.asp. Accessed 10 Nov 2014.

23. Cooper H, Hedges LV, Valentine JC. The handbook of research synthesis and meta-analysis. New York, USA: Russell Sage Foundation; 2009.

24. DerSimonian R, Laird N. Meta-analysis in clinical trials. Control Clin Trials. 1986;7:177-88

25. Higgins J, Thompson SG, Deeks JJ, Altman DG. Measuring inconsistency in meta-analyses. BMJ. 2003;327:557-60.

26. The World Bank Group. Country and Lending Groups. 2015. http://data. worldbank.org/about/country-and-lending-groups. Accessed 4 Mar 2015.

27. StataCorp. Stata Statistical Software: Release 13. College Station, TX: StataCorp LP; 2013.

28. Hoban DJ, Nicolle LE, Hawser S, Bouchillon S, Badal R. Antimicrobial susceptibility of global inpatient urinary tract isolates of Escherichia coli: results from the Study for Monitoring Antimicrobial Resistance Trends (SMART) program: 2009-2010. Diagn Micr Infec Dis. 2011;70:507-11.

29. Kimando JM, Okemo PO, Njagi EN. Resistance to antibiotics in urinopathogenic bacteria isolated in patients attending Kenyatta University Health Clinic, Nairobi. E Afr Med J. 2010;87:115-9.

30. Mwaka AD, Mayanja-Kizza H, Kigonya E, Kaddu-Mulindwa D. Bacteriuria among adult non-pregnant women attending Mulago hospital assessment centre in Uganda. Afr Health Sci. 2011;11:182-9.

31. Prakash D, Saxena RS. Prevalence and antimicrobial susceptibility pattern of Escherichia coli in hospital acquired and community acquired patients related to urinary tract infection in India. J Appl Pharm Sci. 2013;3:124-32.

32. Shaifali I, Gupta U, Mahmood SE, Ahmed J. Antibiotic susceptibility patterns of urinary pathogens in female outpatients. N Am J Med Sci. 2012;4:163-9.

33. World Health Organisation. The evolving threat of antimicrobial resistance: options for action. 2012. http://whqlibdoc.who.int/publications/2012/ 9789241503181_eng.pdf. Accessed 25 Oct 2014.

34. Dalhoff A. Global fluoroquinolone resistance epidemiology and implications for their clinical use. Interdiscip Perspect Infect Dis. 2012; doi:10.1155/2012/976273.

35. Azap OK, Arslan H, Serefhanoglu K, Colakoglu S, Erdogan H, Timurkaynak F, et al. Risk factors for extended-spectrum beta-lactamase positivity in uropathogenic Escherichia coli isolated from community-acquired urinary tract infections. Clin Microbiol Infect. 2010;16:147-51.

36. Hassanzadeh $\mathrm{P}$, Motamedifar M. The prevalence of asymptomatic bacteriuria in long term care facility residents in Shiraz, Southwest Iran: A crosssectional study. Pak J Biol Sci. 2007:10:3890-4.

37. Killgore KM, March KL, Guglielmo BJ. Risk factors for community-acquired ciprofloxacin-resistant Escherichia coli urinary tract infection. Ann Pharmacother. 2004;38:1148-52.

38. Molina-Lopez J, Aparicio-Ozores G, Ribas-Aparicio RM, Gavilanes-Parra S, Chavez-Berrocal ME, Hernandez-Castro R, et al. Drug resistance, serotypes, 
and phylogenetic groups among uropathogenic Escherichia coli including O25-ST131 in Mexico City. J Infect Dec Ctries. 2011;5:840-9.

39. Otajevwo FD. Urinary tract infection among symptomatic outpatients visiting a tertiary hospital based in midwestern Nigeria. Glob J Health Sci. 2013;5:187-99.

40. Sood S, Gupta R. Antibiotic resistance pattern of community acquired uropathogens at a Tertiary Care Hospital in Jaipur, Rajasthan. Indian J Commun Med. 2012;37:39-44.

41. Wang Y, Zhao S, Han L, Guo X, Chen M, Ni Y, et al. Drug resistance and virulence of uropathogenic Escherichia coli from Shanghai. Chin J Antibiot. 2014;67:799-805

42. Ljuca S, Zvizdic S, Hamzic S, Kalajdzija M, Ljuca A. Antimicrobial susceptibility of bacteria isolated from inpatients and outpatients urine samples. Med Arh. 2010;64:161-4

43. Longhi C, Conte MP, Marazzato M, lebba V, Totino V, Santangelo F, et al. Plasmid-mediated fluoroquinolone resistance determinants in Escherichia coli from community uncomplicated urinary tract infection in an area of high prevalence of quinolone resistance. Eur J Clin Microbiol Infect Dis. 2012;31:1917-21.

44. Ni Chulain M, Murray AM, Corbett-Feeney G, Cormican M. Antimicrobial resistance in E. Coli associated with urinary tract infection in the West of Ireland. Ir J Med Sci. 2005;174:6-9.

45. Shariff VAA, Shenoy MS, Yadav T, M R. The antibiotic susceptibility patterns of uropathogenic Escherichia coli, with special reference to the fluoroquinolones. J Clin Diagn Res. 2013;7:1027-30.

46. Al Sweih N, Jamal W, Rotimi VO. Spectrum and antibiotic resistance of uropathogens isolated from hospital and community patients with urinary tract infections in two large hospitals in Kuwait. Med Princ Pract. 2005:14:401-7.

47. Karlowsky JA, Lagacé-Wiens PR, Simner PJ, DeCorby MR, Adam HJ, Walkty A, et al. Antimicrobial resistance in urinary tract pathogens in Canada from 2007 to 2009: CANWARD surveillance study. Antimicrob Agents Chemother. 2011;55:3169-75.

48. Tenover FC. Mechanisms of Antimicrobial Resistance in Bacteria. Am J Med. 2006;119:S3-S10.

49. Maraki S, Mantadakis E, Michailidis L, Samonis G. Changing antibiotic susceptibilities of community-acquired uropathogens in Greece, 2005-2010. J Microbiol Immunol Infect. 2013;46:202-9.

50. Costelloe C, Metcalfe C, Lovering A, Mant D, Hay AD. Effect of antibiotic prescribing in primary care on antimicrobial resistance in individual patients: systematic review and meta-analysis. BMJ. 2010;340:c2096.

51. Vellinga A, Murphy AW, Hanahoe B, Bennett K, Cormican M. A multilevel analysis of trimethoprim and ciprofloxacin prescribing and resistance of uropathogenic Escherichia coli in general practice. J Antimicrob Chemother. 2010;65:1514-20

52. Goettsch W, Van Pelt W, Nagelkerke N, Hendrix M, Buiting A, Petit P, et al. Increasing resistance to fluoroquinolones in Escherichia coli from urinary tract infections in the Netherlands. J Antimicrob Chemother. 2000;46:223-8.

53. Zervos MJ, Hershberger E, Nicolau DP, Ritchie DJ, Blackner LK, Coyle EA, et al. Relationship between fluoroquinolone use and changes in susceptibility to fluoroquinolones of selected pathogens in 10 United States teaching hospitals, 1991-2000. Clin Infect Dis. 2003;37:1643-8.

54. Gottesman BS, Carmeli Y, Shitrit P, Chowers M. Impact of quinolone restriction on resistance patterns of Escherichia coli isolated from urine by culture in a community setting. Clin Infect Dis. 2009:49:869-75.

55. Australian Commission on Safety and Quality in Healthcare. A Report of the Australian One Health Antimicrobial Resistance Colloquium. 2013. https://www.health.gov.au/internet/main/publishing.nsf/Content/ 1803C433C71415CACA257C8400121B1F/\$File/Colloquium-Report-FinalFeb2014.pdf. Accessed 13 Oct 2014.

56. Dancer $\mathrm{SJ}$. Infection control in the post-antibiotic era. Healthc Infect. 2013;18:51-60.

57. Australian Government Department of Health. National Antimicrobial Resistance Strategy 2015-2019. 2015. http://www.health.gov.au/internet/ main/publishing.nsf/Content/1803C433C71415CACA257C8400121B1F/\$File/ amr-strategy-2015-2019.pdf. Accessed 17 Jun 2015.

58. Infectious Diseases Society of the Republic of China. Guidelines for antimicrobial therapy of urinary tract infections in Taiwan. J Microbiol Immunol Infect. 2000;33:271-2.
59. Karaca Y, Coplu N, Gozalan A, Oncul O, Citil BE, Esen B. Co-trimoxazole and quinolone resistance in Escherichia coli isolated from urinary tract infections over the last 10 years. Int J Antimicrob Agents. 2005;26:75-7.

60. Naber KG. Survey on antibiotic usage in the treatment of urinary tract infections. J Antimicrob Chemother. 2000;46:49-52.

61. Olson RP, Haith K. Antibiotic resistance in urinary tract infections in college students. J Am Coll Health. 2012;60:471-4.

62. Okeke IN, Klugman KP, Bhutta ZA, Duse AG, Jenkins P, O'Brien TF, et al. Antimicrobial resistance in developing countries. Part II: strategies for containment. Lancet Infect Dis. 2005;5:568-80.

63. Okeke IN, Lamikanra A, Edelman R. Socioeconomic and behavioral factors leading to acquired bacterial resistance to antibiotics in developing countries. Emerg Infect Dis. 1999:5:18.

64. Ahmad S. Pattern of urinary tract infection in Kashmir and antimicrobial susceptibility. Bangladesh Med Res Counc Bull. 2012;38:79-83.

65. Akoachere JF, Yvonne S, Akum NH, Seraphine EN. Etiologic profile and antimicrobial susceptibility of community-acquired urinary tract infection in two Cameroonian towns. BMC Res Notes. 2012;5:219.

66. Akram M, Shahid M, Khan AU. Etiology and antibiotic resistance patterns of community-acquired urinary tract infections in I N M C Hospital Aligarh, India. Ann Clin Microbiol Antimicrob. 2007; doi:http://dx.doi.org/10.1186/ 1476-0711-6-4

67. Arabi FMZ, Banazadehi A. Prevalence and antimicrobial susceptibility patterns of uropathogens among patients referring to valieasr laboratory in Najafabad, Isfahan. Iran Mid East J Sci Res. 2013;13:85-90.

68. Astal ZE. Increasing ciprofloxacin resistance among prevalent urinary tract bacterial isolates in the Gaza Strip. Singapore Med J. 2005;46:457-60.

69. Dash M, Padhi S, Mohanty I, Panda P, Parida B. Antimicrobial resistance in pathogens causing urinary tract infections in a rural community of Odisha. India J Family Community Med. 2013;20:20-6.

70. Kashef N, Djavid GE, Shahbazi S. Antimicrobial susceptibility patterns of community-acquired uropathogens in Tehran. Iran J Infect Dev Ctries. 2010; 4:202-6.

71. Kothari A, Sagar V. Antibiotic resistance in pathogens causing communityacquired urinary tract infections in India: a multicenter study. J Infect Dev Ctries. 2008;2:354-8.

72. Murugan K, Savitha T, Vasanthi S. Retrospective study of antibiotic resistance among uropathogens from rural teaching hospital, Tamilnadu. India Asian Pac J Trop Dis. 2012;2:375-80

73. Sire JM, Nabeth P, Perrier-Gros-Claude JD, Bahsoun I, Siby T, Macondo EA, et al. Antimicrobial resistance in outpatient Escherichia coli urinary isolates in Dakar. Senegal J Infect Dev Ctries. 2007;1:263-8.

74. Morgan DJ, Okeke IN, Laxminarayan R, Perencevich EN, Weisenberg S. Nonprescription antimicrobial use worldwide: a systematic review. Lancet Infect Dis. 2011;11:692-701.

75. Dalhoff A. Resistance surveillance studies: a multifaceted problem—the fluoroquinolone example. Infection. 2012;40:239-62.

76. Maron DF, Smith TJ, Nachman KE. Restrictions on antimicrobial use in food animal production: an international regulatory and economic survey. Global Health. 2013;9:48.

77. Owumi W, Banaei N, Shortliffe LD. Adult and Pediatric Intra-Institutional Trends of Ciprofloxacin Susceptibility in E. coli Positive Urinary Cultures. Antibiotics. 2014;3:163-73.

78. Storby KA, Österlund A, Kahlmeter G. Antimicrobial resistance in Escherichia coli in urine samples from children and adults: a 12 year analysis. Acta Paediatr. 2004;93:487-91.

79. Adefurin A, Sammons H, Jacqz-Aigrain E, Choonara I. Ciprofloxacin safety in paediatrics: a systematic review. Arch Dis Child. 2011;96:874-80.

80. Von Elm E, Egger M. The scandal of poor epidemiological research: reporting guidelines are needed for observational epidemiology. BMJ. 2004; 329:868.

81. Vandenbroucke JP, Von Elm E, Altman DG, Gøtzsche PC, Mulrow CD, Pocock SJ, et al. Strengthening the Reporting of Observational Studies in Epidemiology (STROBE): explanation and elaboration. Ann Intern Med. 2007; 147:W-163-W-94. 